\title{
Cognitive impact in children with "benign" childhood focal epilepsy with centrotemporal spikes
}

\author{
Natalie Helene van Cleef Banaskiwitz¹, Carmen Silvia Molleis Galego Miziara², Alana Batista Xavier3, Maria Luiza \\ Giraldes de Manreza², Alisson Paulino Trevizol ${ }^{4}$, Álvaro Machado Dias ${ }^{4}$, Antonio de Pádua Serafim55,6
}

1 PhD Student on the Neurosciences and Behavior Program, Institute of Psychology, University of São Paulo (USP), São Paulo, SP, Brazil. 2 Department of Neurology, University of São Paulo School of Medicine (FMUSP), São Paulo, SP, Brazil.

${ }_{3}$ Postgraduate Neurology Program, Health Sciences Center, Universidade Federal do Estado do Rio de Janeiro (UNIRIO), Rio de Janeiro, RJ, Brazil.

${ }^{4}$ Center for Neuromodulation Studies, Department of Psychiatry of the Federal University of São Paulo Medical School (Unifesp), São Paulo, SP, Brazil.

5 Department of Psychiatry, University of São Paulo School of Medicine (FMUSP), São Paulo, SP, Brazil.

${ }_{6}$ Methodist University of São Paulo (Umesp), São Bernardo do Campo, SP, Brazil.

Received: 03/27/2017 - Accepted: 06/08/2017

DOI: 10.1590/0101-60830000000129

\begin{abstract}
Background: Cognitive alterations are associated with benign childhood focal epilepsy with centrotemporal spikes (BCECTS) including aspects of executive functions. Objectives: This study presents the performance profile on attention and executive function tests of fifty-eight children (BCECTS, $n=30$ and controls, $\mathrm{n}=28$ ) aged 8-13 years. Methods: The following tools were employed: Vocabulary and Block Design subtests from the Wechsler Intelligence Scale for Children III, Stroop Test, Modified Card Sorting Test, Controlled Oral Word Association - FAS and Tower of London. Results: Children with BCECTS presented average IQ measure, although their performance was statistically worse when compared to the control group. Children with BCECTS showed significantly lower performance compared to the control group in the following variables: total number of recollected words on the oral fluency test, total number of categories, categorization effect and total number of errors in MCST; and execution time for the Stroop Test Card 1. After controlling for the IQ effect, the total number of errors in the MCST did not show any significant difference between the groups. Discussion: Children with BCECTS showed lower performance in attention and executive functions when compared to healthy children. The results suggest that the concept of "benign" BCECTS should be reconsidered.
\end{abstract}

Banaskiwitz NHC et al. / Arch Clin Psychiatry. 2017;44(4):99-102

Keywords: Childhood epilepsy, cognition, executive function, attention, child psychiatry.

\section{Introduction}

Benign focal childhood epilepsy with centrotemporal spikes (BCECTS) is one of the most frequent epileptic syndromes in childhood. Given its favorable prognosis, with seizures generally ceasing near puberty with normalization of electroencephalogram (EEG), neuropsychological evaluation was usually not considered. However, reports on cognitive disturbances presented by children with BCECTS have challenged the concept of favorable prognoses. Executive and attentional deficits ${ }^{1}$, related to daily activities and proper social relationships have been previously described ${ }^{1-11}$, including reading disabilities, sustained attention deficits, selective attention deficits, divided attention deficits, visuomotor and behavior difficulties.

The sustained attention deficits may be related to rightsided epileptiform activities in BCECTS, interfering in the right hemisphere function of sustaining attention. Attention is involved in cognitive functions and enables access to memory, sensory stimuli and motor responses.

These functions are acquired gradually during childhood and adolescence ${ }^{12}$, progressively providing the child with the abilities to initiate, persist and accomplish tasks ${ }^{13}$. This study aims to investigate the performance profile of children with BCECTS by using neuropsychological tests that evaluate cognitive domains such as attention and executive functions and compare them to the performance of children with no neurological disturbances. We compared the differences in attention and executive functions between the performance of children with BCECTS and children without epilepsy.

\section{Methods}

\section{Participants}

Fifty-eight children aged between 8 to 13 took part of this study. Thirty children fulfilled the clinical and electroencephalographic criteria for BCECTS, according to the International League Against the Epilepsy (ILAE). All the children from the study group were assisted at the Child Neurology Clinic at the Hospital das Clínicas at the Universidade de São Paulo. The exclusion criteria were: estimated Intellectual Quotient (IQ) < 70; neuroimaging abnormalities; clinical disorders interfering with cognitive abilities; diagnosis of attention deficit hyperactivity disorder; and medical treatment for psychiatric and/or other central nervous system (CNS) disorders (except primary headache).

The control-group was represented by twenty-eight children according to demographic variables: age, gender, schooling and socio-economic levels, and selected among students in São Paulo city public schools. The exclusion criteria were the same employed for BCECTS children, in addition to the lack of complaints regarding learning difficulties. The groups were similar according to gender, age, schooling and socio-economic status.

\section{Procedure}

All children with BCECTS were submitted to a neurological evaluation and complementary exams, neuroimaging, cranial tomography (CT) or cranial magnetic resonance (MR) and EEG. Both study and control groups were submitted to the same neuropsychological evaluation. 
A questionnaire assessing the socio-demographic data was applied to the responsible informants. In order to check for hand preference, the child was given a pencil and a sheet of paper and asked to write his/her name. The hand spontaneously chosen to accomplish the task was recorded. The study was approved by the Ethics Committee of the University of São Paulo Medical School and written informed consent was obtained from respective responsible persons.

\section{Measures (neuropsychological evaluation) and instruments}

Intellectual efficiency: short form of the Wechsler Intelligence Scale for Children Third Edition ${ }^{14,15}$, i.e., Vocabulary and Block Design subtests sum of the scaled scores for the calculation of estimated IQ ${ }^{16}$.

Attention and executive functions: Modified Card Sorting Test ${ }^{17}$, to evaluate the executive functions of cognitive flexibility, categorization ability and attention errors in the strategy for action; Controlled Oral Word Association - FAS 18 used as a measurement of phonemic verbal fluency; Victoria Stroop Test ${ }^{19,20}$, to evaluate selective attention and mental flexibility; Tower of London ${ }^{21}$ as a measurement of the executive planning function.

\section{Statistical analysis}

The results were analyzed with the statistical package STATA/SE version 11 for Windows. The chi-squared test for categorical variables and the T-test for numerical variables analysis were performed to verify the differences between control and study groups concerning the socio-demographic variables and the tests scores. When the groups presented different variances, the unequal variance t-test was used and if the groups presented equivalent variances, the t-test for equal variances was chosen. The differences between the control and study groups concerning the estimated IQ were also evaluated by the t-test. Further analyses were made controlling for the IQ measure to verify the significance of the cognitive variables. In order to test the influence of the estimated IQ in each test score presenting difference (dependent variables) between the groups (independent variable), the covariance analysis (ANCOVA) was used, isolating the IQ interference (covariable) and checking if the difference between the groups persisted. Furthermore, the relationship between the estimated IQ and the scores of the tests for attention and executive function was verified by Pearson correlation. The significance level established was $\mathrm{P} \leq 0.05$.

\section{Results}

Fifty-eight children were evaluated ( 32 boys and 26 girls) between the ages of 8 and 13 (average age $=10.3 \mathrm{SD}=1.7$ ), all of them from public schools in the city of São Paulo. Among these, thirty children ( 18 boys and 12 girls) with an average age of $10.5(\mathrm{SD}=1.7)$ and diagnosed with BCECTS, represent the study-group and twenty eight healthy children (14 boys and 14 girls) with an average age of $10.11(\mathrm{SD}=1.7)$ represented the control-group.

Regarding the laterality of the epileptiform activity in the EEG, ten children (70\% male) presented left-sided discharges, 9 children (78\% male) right-sided discharges and eight children (75\% female) presented bilateral discharges. Regarding the use of anticonvulsants, fourteen children $(71 \%$ male) were not taking medication while thirteen (62\% female) were under medication, of which twelve were under monotherapy and only one under polytherapy. Of the 30 children, three showed no epileptiform activity on EEG.

The comparative statistical analysis between the study and the control groups showed that the groups were homogeneous regarding socio-demographic variables.

\section{Intellectual efficiency}

The comparison between the estimated IQ in both groups showed a significant difference between the means $(p=0.013)$ and a better efficiency in the control group (mean $=112.28 ; \mathrm{SD}=2.20$ ) in contrast to the study group $($ mean $=103.13 ; \mathrm{SD}=2.76)$ as shown in Table 1 . The analysis of the relationship between the estimated IQ and the efficiency in the tests showed significant associations only for some of the test scores. Also, despite presenting a weak correlation, the IQ results were positively correlated with the total number of words in FAS (rho $=0.392$ ).

\section{Attention and executive functions}

\section{Modified Card Sorting Test}

The groups were statistically different when comparing the number of categories score $(p=0.007)$, categorization efficiency $(p=0.007)$ and total errors $(p=0.019)$ revealing a better performance of the controlgroup, as shown in Table 1. There were no significant difference in the number of perseverative errors and failure to maintain set.

\section{Controlled Oral Word Association - FAS}

The analysis of the results revealed significant difference $(\mathrm{p}=$ 0.0004 ) between the groups for the Verbal Fluency test with a better performance of the control group as shown in Table 1 .

\section{Victoria Stroop Test}

The analysis of the execution time in boards 1 and 3 and errors in board 3 as well as time of board 2 in the Stroop test - Victoria version showed significant differences only for the time of board 1 of the test $(\mathrm{p}=0.029)$.

Due to the fact that one subject of the study group could not read, therefore could not run the Cards 1 and 2, a separate analysis was conducted for this test. It is presented in Table 1 .

Table 1. Mean (SD) neuropsychological test results for the study group and control group

\begin{tabular}{|c|c|c|c|}
\hline Measure & BCECTS & Control Group & $p$ \\
\hline${ }^{*} \mathrm{IO}$ & $103.13(15.12)$ & $112.28(11.68)$ & 0.013 \\
\hline${ }^{*} \mathrm{MCST}$ - No. of categories & $3.8(1.64)$ & $4.85(1.17)$ & 0.007 \\
\hline *MCST - Categorization efficiency & $23.86(11.33)$ & $31.75(10.20)$ & 0.007 \\
\hline${ }^{*}$ MCST - Total errors & $16.36(7.58)$ & $11.5(7.79)$ & 0.019 \\
\hline${ }^{*}$ MCST - Perseverative errors & $4.16(3.21)$ & $2.82(3.25)$ & 0.119 \\
\hline${ }^{*}$ MCST - Set loss & $0.67(0.08)$ & $0.67(0.94)$ & 0.510 \\
\hline${ }^{*}$ F.A.S. & $16.93(6.54)$ & $23.5(6.77)$ & 0.0004 \\
\hline${ }^{* *}$ Stroop Test I & $20.17(6.93)$ & $16.82(3.92)$ & 0.029 \\
\hline${ }^{*}$ Stroop Test II & $25.56(7.59)$ & $24.35(7.73)$ & 0.461 \\
\hline **Stroop Test III & $36.89(2.73)$ & $34.71(1.92)$ & 0.519 \\
\hline${ }^{* *}$ Stroop Test III errors & $0.62(1.11)$ & $0.32(0.47)$ & 0.193 \\
\hline${ }^{*}$ Tower of London - total score & $28.46(4.00)$ & $20.03(3.31)$ & 0.111 \\
\hline${ }^{*}$ Tower of London - extra attempts & $6.6(3.05)$ & $5.57(2.94)$ & 0.198 \\
\hline${ }^{*}$ Tower of London - planning time & $6.79(5.13)$ & $5.09(3.68)$ & 0.156 \\
\hline *Tower of London - mean execution time & 24.52 (12.44) & $19.65(8.65)$ & 0.091 \\
\hline
\end{tabular}

* T-test for equalvariances. ${ }^{* *} \mathrm{~T}$-test for unequalvariances.

\section{Tower of London}

There were no significant differences for the total score, number of extra attempts, mean time of planning and execution between both groups. The values are described in Table 1.

\section{Differences between groups in attention and executive function tests after isolating of the 10 interference}

ANCOVAs of the group effect for the scores in Stroop Test, MCST and FAS setting estimated IQ as a covariant revealed significant 
differences for most of the considered tests, except for the Total errors score in the MCST, as shown in Table 2.

Table 2. Result of ANCOVAs on the group effect over the scores in Stroop, MCST and FAS tests setting estimated $\mathrm{Ql}$ as covariant

\begin{tabular}{|l|l|c|c|}
\hline Test & Variable & $F$ & $p$ \\
\hline Stroop & Execution time for board & 4.48 & 0.039 \\
\hline \multirow{3}{*}{ MCST } & No. of categories & 5.09 & 0.028 \\
\cline { 2 - 4 } & Categorization efficiency & 4.98 & 0.030 \\
\cline { 2 - 4 } & Total errors & 3.70 & 0.060 \\
\hline \multirow{2}{*}{ FAS } & Recollected words with the letters F, A and S & 8.66 & 0.005 \\
\hline
\end{tabular}

\section{Discussion}

This study aimed to verify the performance obtained by children with benign focal epilepsy in childhood and children without epilepsy in neuropsychological tests of attention and executive functions. Studies have shown an association between neurological disorders and cognitive deficits ${ }^{1-10}$.

Although children with BCECTS presented average levels of estimated IQ, their performance was significantly lower in specific attention and executive functions tasks compared to that obtained by the control group. This result is similar to the ones presented by other studies $2,5,10,22-25$. It is noteworthy that most of these studies used all or at least eight of the Wechsler Intelligence scale subtests for the calculation of the total IQ, while we used only two subtests (Block Design and Vocabulary) for the estimated IQ calculation. The choice for the short form to estimate intellectual efficiency did not compromise the analysis of results because it took into account the reliability of both subtests as well as its correlation to the Global Scale of the WISC-III. Both Vocabulary and Block Design subtests present a high correlation with the global scale and provide good measurements to evaluate general intelligence ${ }^{14,16}$. On the other hand, some authors did not show any differences between the IQ of BCECTS children and children in the control groups $1,2,6,9,23,26-28$.

This study showed that children with BCECTS presented significantly worse performance than the control group in executive functions of cognitive flexibility, categorization ability and attention errors in the strategy for action, in phonemic verbal fluency and selective attention and mental flexibility. Notwithstanding, the performance in executive planning function did not present discrepancy in comparison with the control group.

The results from the Victoria Stroop evaluating selective attention and mental flexibility suggests that children with BCECTS present an adequate ability for selective attention and mental flexibility. However, slower performance in naming colors, which correlates with previous studies finding that children with BCECTS perform with reduced speed in attention tasks, was also observed ${ }^{29}$. However, studies using other versions of the Stroop test showed different results. Chevalier et al. ${ }^{4}$ found a significantly higher number of errors made by children with BCECTS with the Incongruous card and no difference between the groups concerning the performance with the Control card. Baglietto et al. ${ }^{5}$ showed execution time significantly higher in the study group compared to the control group, in addition to a higher number of errors in the first two cards. In a more recent study, the discrepancy among the results can be explained by the use of different modalities of the Stroop test. However, these results suggest that the deficient performance of children with BCECTS is more likely related to the precision (measured by the total of errors) than to the response speed.

Difficulties in planning and problem solving as assessed by the Tower of London were not found in our study, contrary to previous data showing worse performances for the children with BCECTS compared to the control-group ${ }^{1}$.

The Phonemic Fluency Test (Controlled Oral Word Association) seems to be sensitive to the presence of BCECTS. Studies showed that children with BCECTS present worse performances in phonemic verbal fluency tasks 1,22 even after total remission of seizures and EEG normalization $5,9,10$. Such results agree with the results obtained in this study and seem to confirm the presence of alterations in the verbal fluency in children with BCECTS.

Finally, although we used the Modified WCST, differences in performance in the MCST for executive functions of cognitive flexibility, categorization ability and attention errors in the strategy for action were verified. The comparison between groups revealed worse performance in number of categories, categorization efficiency and total number of errors scores for children with BCECTS, however no significant difference related to perseverative errors and inability to maintain the set. Our results partially agree with the results presented by Hoie et al. ${ }^{22}$, which showed lower performance of children with BCECTS in all WCST scores compared to the control-group.

Although our study has showed worse performance of children with BCECTS in executive functions of cognitive flexibility, categorization ability and attention errors in the strategy for action, in phonemic verbal fluency and selective attention and mental flexibility, the impact of the intellectual functions in the performance of these tests must be considered, since there were differences in the estimated IQ between the groups. The analysis of correlations between the tests and the estimated IQ showed weak or null correlations between some of the tests' scores. However, studies with healthy children showed that the estimated IQ influences the performance on executive functions tests such as WCST and the Phonemic Fluency Test ${ }^{30}$. This issue is usually neglected in studies $5,9,23,25,27$ or is approached as an attempt to match the study and control groups according to the participants IQ8,10,11,22,29.

This study has some limitations. One of them is that we did not assess the possible influence of the frequency of epileptiform activity on cognitive functioning, as many studies have linked the higher frequency of discharges with an increased cognitive loss $2,22,25$. The fact that previous studies used different instruments for the assessment of cognitive functioning, and that only a few of them included executive functions instruments, do not allow further comparisons. In addition, a possible limitation is that psychiatric symptoms were not actively asked.

\section{Conclusion}

Our findings show that children with BCECTS present impaired categorization ability, worse performance in color naming and deficits in verbal fluency. These cognitive abnormalities are not influenced by the intellectual capacity. Another important aspect is that, despite evidence that BCECTS do impact on cognition, we must consider that nearly half of the sample was taking antiepileptic drugs, which probably plays an influence the cognition of children.

\section{References}

1. Croona C, Kihlgren M, Lundberg S, Eeg-Olofsson O, Eeg-Olofsson KE. Neuropsychological findings in children with benign childhood epilepsy with centrotemporal spikes. Dev Med Child Neurol. 1999;41:813-8.

2. Weglage J, Demsky A, Pietsh M, Kurlemann G. Neuropsychological, intellectual, and behavioral findings in patients with centrotemporal spikes with and without seizures. Dev Med Child Neurol. 1997;39:646-51.

3. Gündüz E, Demirbilek V, Korkmaz B. Benign rolandic epilepsy: neuropsychological findings. Seizure. 1999;8:246-9.

4. Chevalier H, Metz-Lutz MN, Segalowitz SJ. Impulsivity and control of inhibition in Benign Focal Childhood Epilepsy (BFCE). Brain Cogn. 2000;43:86-9.

5. Baglietto MG, Battaglia FM, Tortonelli S, De Negri E, Calevo MG, Veneselli E. Neuropsychological disorders related to interictal epileptic discharges during sleep in benign epilepsy of childhood with centrotemporal or rolandic spikes. Dev Med Child Neurol. 2001;43:407-12.

6. D’Alessandro P, Piccirilli M, Ibba A, Maiotti M, Sciarma T. Neuropsychological features of benign Partial epilepsy in children. Ital J Neurol Sci. 1990;11:265-9.

7. Pinton F, Ducot B, Mott J, Arbues AS, Barondiot C, Barthez MA, et al. Cognitive functions in children with benign childhood epilepsy with centrotemporal spikes. Epileptic Disord. 2006;8(1):11-23. 
8. Cerminara C, D’Agati E, Lange KW, Kaunzinger I, Tucha O, Parisi P, et al. Benign childhood epilepsy with centrotemporal spikes and the multicomponent model of attention: a matched control study. Epilepsy Behav. 2010;19(1):69-77.

9. Lindgren A, Kihlgren M, Melin 1, Croona C, Lundberg S, Eeg-Olofson O. Development of cognitive functions in children of rolandic epilepsy. Epilepsy Behav. 2004;5(6):903-10.

10. Riva D, Vago C, Franceschetti S, Pantaleoni C, D'Arrigo S, Granata T, et al. Intellectual and language findings and their relationship to EEG characteristics in benign childhood epilepsy with centrotemporal spikes. Epilepsy Behav. 2007;10:278-85.

11. Titomanlio L, Romano A, Romagnuolo G, Del Giudice E. Subtle neuropsychological alterations in children with rolandic epilepsy. Ital J Pediatr. 2003;29:276-80.

12. Anderson V. Assessing executive functions in children: biological, psychological, and developmental considerations. Pediatr Rehabil. 2001;4(3):119-36.

13. Blair C. School readiness: integrating cognition and emotion in a neuropsychological conceptualization of children's functioning at school entry. American Psychol. 2005;57(2):111-27.

14. Wechsler D. WISC III Manual. Canadian supplement. Toronto: Psychological Corporation; 1996.

15. Figueiredo VLM. Escala de Inteligência Weshsler para Crianças. 3a ed. São Paulo: Casa do Psicólogo Editora Ltda.; 2002.

16. Spreen O, Strauss E. A compendium of neuropsychological tests: administration, norms and commentary. 2nd ed. New York: Oxford University Press; 1998.

17. Nelson HE. A modified card sorting test sensitive to frontal lobe defects. Cortex. 1976;12:313-24.

18. Benton AL, Hamsher KD. Multilingual aphasia examination. Iowa City: AJA Associates; 1989.

19. Stroop JP. Studies of interference in serial verbal reactions. J Exp Psychol. 1935;18:643-62.
20. Miyake A, Friedman NP. The Nature and Organization of Individual Differences in Executive Functions: Four General Conclusions. Curr Dir Psychol Sci. 2012;21(1):8-14.

21. Krikorian R, Bratok J, Gray N. Tower of London Procedure: A Standard Method and Developmental Data. J Clin Exp Neuropsychol. 1994;16(6):840-50

22. Hoie B, Mykletun A, Waaler PE, Skeidsvoll H, Sommerfelt K. Executive function and seizure-related factors in children with epilepsy in western Norway. Dev Med Child Neurol. 2006;48:519-25.

23. Fonseca LC, Tedrus GMAS, Pacheco EMC. Epileptiform EEG discharges in benign childhood epilepsy with centrotemporal spikes: reactivity and transitory cognitive impairment. Epilepsy Behav. 2007;11(1):65-70.

24. Northcott E, Connoly AM, Berroya A, Sabaz M, McIntyre J, Christie J, et al. The neuropsychological and language profile of children with centrotemporal benign rolandic epilepsy. Epilepsia. 2005;46(6):924-30.

25. Goldberg E, Bilder RM. The frontal lobes and hierarchical organization of cognitive control. In: Perecman E (Ed). The frontal lobes revised. New York: JRBN Press; 1987. p. 159-87.

26. Giordani B, Caveney AF, Laughrin D, Huffman JL, Berent S, Sharma $\mathrm{U}$, et al. Cognition and behavior in children with benign epilepsy with centrotemporal spikes (BECTS). Epilepsy Res. 2006;70:89-94.

27. Ay Y, Gokben S, Serdaroglu G, Polat M, Tosun A, Tekgul H, et al. Neuropsychologic Impairment in Children With Rolandic Epilepsy. Pediatr Neurol. 2009;41(5):359-63.

28. Tedrus GMAS, Fonseca LC, Castilho DP, Pacheco EMC, Campregher AA, Bittar MC. Benign childhood epilepsy with centro-temporal spikes. Evolutive clinical, cognitive and EEG aspects. Arq Neuropsiquiatr. 2010;68(4):550-5.

29. Deltour L, Querné L, Vernier MP, Berquin P. Deficit of endogenous spatial orienting of attention in children with benign epilepsy with centrotemporal spikes (BECTS). Epilepsy Res. 2008;79:112-9.

30. Arffa S. The relationship of intelligence to executive function and nonexecutive function measures in a sample of average, above average, and gifted youth. Arch Clin Neuropsychol. 2007;22:969-78. 